This item was submitted to Loughborough's Research Repository by the author.

Items in Figshare are protected by copyright, with all rights reserved, unless otherwise indicated.

\title{
Twist limits for late twisting double somersaults on trampoline
}

PLEASE CITE THE PUBLISHED VERSION

http://dx.doi.org/10.1016/j.jbiomech.2017.05.002

\section{PUBLISHER}

(c) Elsevier

VERSION

AM (Accepted Manuscript)

\section{PUBLISHER STATEMENT}

This work is made available according to the conditions of the Creative Commons Attribution-NonCommercialNoDerivatives 4.0 International (CC BY-NC-ND 4.0) licence. Full details of this licence are available at: https://creativecommons.org/licenses/by-nc-nd/4.0/

\section{LICENCE}

CC BY-NC-ND 4.0

\section{REPOSITORY RECORD}

Yeadon, Fred, and Michael Hiley. 2017. "Twist Limits for Late Twisting Double Somersaults on Trampoline". Loughborough University. https://hdl.handle.net/2134/25916. 
Journal of Biomechanics 58, 174-178, 2017

\title{
Twist limits for late twisting double somersaults on trampoline
}

\author{
M.R. Yeadon and M.J. Hiley \\ School of Sport, Exercise and Health Sciences, Loughborough University, Loughborough LE11 3TU, UK
}

\begin{abstract}
An angle-driven computer simulation model of aerial movement was used to determine the maximum amount of twist that could be produced in the second somersault of a double somersault on trampoline using asymmetrical movements of the arms and hips. Lower bounds were placed on the durations of arm and hip angle changes based on performances of a world trampoline champion whose inertia parameters were used in the simulations. The limiting movements were identified as the largest possible odd number of half twists for forward somersaulting takeoffs and even number of half twists for backward takeoffs. Simulations of these two limiting movements were found using simulated annealing optimisation to produce the required amounts of somersault, tilt and twist at landing after a flight time of $2.0 \mathrm{~s}$. Additional optimisations were then run to seek solutions with the arms less adducted during the twisting phase. It was found that $3 \frac{1}{2} 2$ twists could be produced in the second somersault of a forward piked double somersault with arms abducted $8^{\circ}$ from full adduction during the twisting phase and that three twists could be produced in the second somersault of a backward straight double somersault with arms fully adducted to the body. These two movements are at the limits of performance for elite trampolinists.
\end{abstract}

Keywords: computer simulation, aerial movements, technique

\section{INTRODUCTION}

Predicting limiting performances in a sport can be problematic when the limiting factors are primarily physiological. For example, quantifying the lowest possible 100 metre sprint time is fraught with difficulty since the minimum time is more dependent upon the optimal physical attributes of an individual than inherent mechanical constraints. In contrast aerial movements are largely constrained by mechanical factors rather than by individual physical limitations. In addition the measure of sprint time is a continuous variable whereas the number of somersaults and twists are discrete measures. In double somersaults with twist the amount of twist will be a whole number of half twists. On trampoline double somersaults which initially rotate forward will have an odd number of half twists while those initially rotating backward will have an even number of half twists. As a consequence the final direction of somersault rotation is backward which allows viewing of the trampoline bed prior to landing and permits adjustments to be made at the end of the aerial phase and during the takeoff for the next movement.

In a double somersault twist may be confined to just one somersault or may occur in both somersaults. When there is twist in the first somersault there are typically contributions from both contact and aerial twisting techniques (Yeadon, 1993a, b, c, d). When there is no twist in the first somersault, aerial techniques are responsible for the production of twist during flight. This study will investigate the limits of aerial techniques for producing twist and will be confined to double somersaults with the twist in the second somersault since these movements must employ aerial twist and do not have a contact twist contribution. 
Aerial twist in somersaults with multiple twists is a consequence of producing tilt of the longitudinal axis away from the vertical somersault axis using asymmetrical movements of the arms or hips (Yeadon, 1993c, d). The amount of tilt produced may be enhanced by the nutation effect in which the tilt angle increases during the first quarter twist if the arms are abducted away from the body (Yeadon, 1993a, c). For a recorded performance the computer simulation model of Yeadon et al. (1990) may be used to partition the production of tilt into contributions from contact and aerial techniques (Yeadon, 1993d).

In previous research on limiting movements using computer simulation models Hiley and Yeadon (2005) showed that it was theoretically possible to perform a triple straight backward somersault dismount from high bar, providing the release could be timed to within $28 \mathrm{~ms}$. Brüggemann and Arampatzis (1993) determined that a quadruple tucked backward somersault dismount was possible in principle. King and Yeadon (2004) found that large linear and angular approach velocities were key for maximising rotation in tumbling and that a straight triple somersault should be possible. A straight triple somersault has now been performed in tumbling competition. In gymnastics vaulting it was found that a handspring double somersault with $1 \frac{1}{2}$ twists and a handspring triple somersault tucked were limiting vaults (Hiley et al., 2015). A handspring triple tucked somersault vault has now been attempted in competition. In triple somersaults in the aerials event of freestyle skiing, six twists will be the limit according to Yeadon (2013).

The aim of this research study was to determine the twist limits for double somersaults with the twist in the second somersault. Since aerial twist may be produced using asymmetrical arm or asymmetrical hip movements it will be of interest to determine the individual tilt contributions for the limiting movements.

\section{METHODS}

An angle-driven computer simulation model of aerial movement (Yeadon, 1990a; Yeadon et al., 1990) was used to determine the limits of asymmetrical arm and hip techniques for producing aerial twist in the second somersault of a double somersault using the segmental inertia parameters of a male world trampoline champion (Yeadon, 1990b). The model comprised 11 segments and required the initial angular momentum and body orientation as input together with the time histories of the joint angles. Elbow and knee flexion were not used and neither was relative movement of the shoulder girdle. As a consequence the model was reduced to six segments: chest + head, pelvis, two legs and two arms. Side flexion was shared between the hips and the spine as was hyperextension whereas forward flexion occurred solely at the hip joints for the first $90^{\circ}$ of flexion and thereafter was shared between the hips and spine (Yeadon, 1990c). In addition the two legs moved together so that the six degrees of freedom at the hip joints and spine became two independent degrees of freedom. Constant angular momentum during flight was assumed and the equations of motion were solved numerically for whole body angular velocity from which somersault, tilt and twist angles were obtained by numerical integration. Somersault gave the whole body rotation about the (horizontal) angular momentum vector, tilt gave the angle between the longitudinal axis and the vertical plane perpendicular to the angular momentum vector, and twist gave the rotation about the longitudinal axis. The model was evaluated by comparing the twist angles from simulation with five performances of single and double somersaults with twist performed by the aforementioned world trampoline champion: differences were 
less than 0.04 revolutions of somersault and 0.12 revolutions of twist (Yeadon et al., 1990).

In multiple somersaults with multiple twists, the number of twists that can be achieved is limited by the time that the body can be extended in a straight position and so, in general, flight time and somersault momentum will be limiting factors. On trampoline flight time has an upper limit of around $2.0 \mathrm{~s}$ and it is possible to perform a triple straight somersault which will have 50\% more angular momentum than a double straight somersault. As a consequence flight time was set at $2.0 \mathrm{~s}$ in this study and no specific constraints were needed to limit angular momentum.

The model was used to simulate the aerial phase of double somersaults in which twist was initiated at the end of the first somersault and stopped at the end of the second somersault using asymmetrical movements of the arms and hips to produce tilt away from the vertical somersault plane and subsequently to remove it. The maximum amounts of twist in the first $1.5 \mathrm{~s}$ during which tilt is produced and in the last $0.5 \mathrm{~s}$ during which tilt is removed were added together to determine a limiting movement with the maximum number of half twists. An optimised simulation was then found in which the target angles of somersault, tilt and twist were met. Details are given in the following paragraphs.

Seven constraints were imposed when producing a simulation: (a) at the 1.0 somersault position the twist was not more than 0.25 revolutions, (b) the final twist was an odd number of half twists for forward rotating takeoffs and was an even number of half twists when the initial direction of somersault was backward, (c) the final somersault angle gave a landing on the feet with the legs close to vertical, (d) the final tilt angle was zero, (e) arm abduction was restricted to be a maximum of $90^{\circ}$ during the initiation of twist, (f) arm abduction angles were between $90^{\circ}$ and $180^{\circ}$ (hands higher than shoulders) and were symmetrical at the end of the simulation, $(\mathrm{g})$ the time of flight was $2.0 \mathrm{~s}$. Constraint (e) was used in order to follow current trampolining technique and to avoid sequential arm movements of large amplitude. Constraint (f) was used since the arms are typically raised overhead during landing.

Two cases were considered. In the first case asymmetrical hip movement was used to move from $60^{\circ}$ forward flexion to $60^{\circ}$ side flexion from a piked position in a forward rotating double somersault. In the second case asymmetrical hip movement was used to move from a straight position to $30^{\circ}$ side flexion in a straight backward rotating double somersault.

Each change in joint angle was specified by the start and end angle values and the start and end times and was effected using a quintic function with zero velocity and acceleration at the endpoints (Hiley and Yeadon, 2003). Lower limits on the duration of arm and hip movements were based on times between angle turning points in recorded performances of twisting double somersaults by the world trampoline champion whose segmental inertias were used in simulations. For arm abduction through $180^{\circ}$ a minimum duration of $0.30 \mathrm{~s}$ was imposed while $0.20 \mathrm{~s}$ was used for a $90^{\circ}$ arm movement. For $90^{\circ}$ hip flexion / extension a lower limit of $0.25 \mathrm{~s}$ was set and $0.20 \mathrm{~s}$ was used for a change from $60^{\circ}$ hip flexion to $60^{\circ}$ side flexion (a change in hula angle of $90^{\circ}$ ).

In order to maximise the amount of twist produced, the timings of the arm and hip movements were adjusted to maximise the amount of tilt achieved in the second somersault. After an initial side flexion with both arms abducted at $90^{\circ}$, one arm was adducted to the side of the body and as the quarter twist position was reached, the body was straightened and the other arm adducted to the body (Figures 1, 2). The 
majority of the twist then occurred during this twisting phase in a fixed body configuration. Finally the timings of the asymmetrical arm and hip movements, along with the value of the final common arm abduction angle, were used to remove the tilt and stop the twist prior to landing.
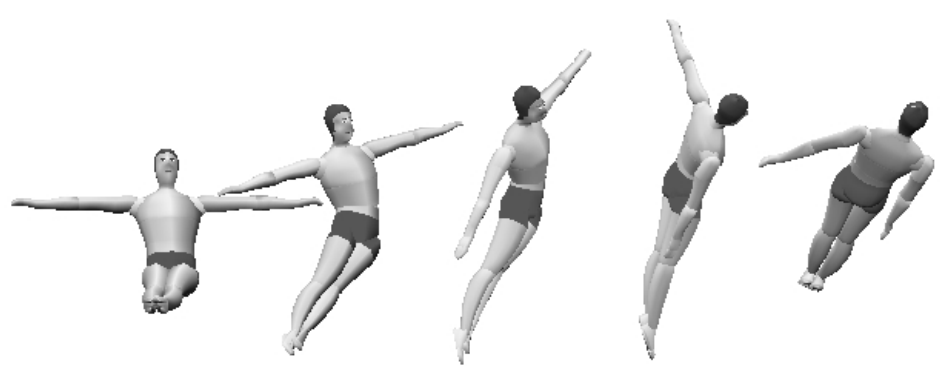

Figure 1. Asymmetrical hip and arm movements used to produce tilt in a piked double forward somersault at the start of the second somersault (front view).
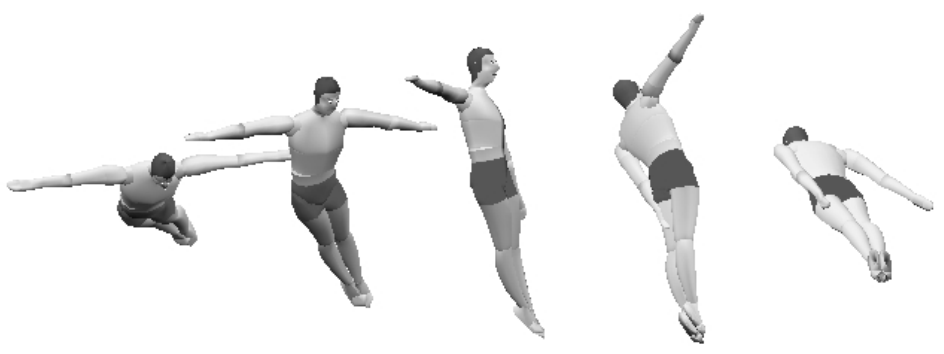

Figure 2. Asymmetrical hip and arm movements used to produce tilt in a straight double backward somersault at the start of the second somersault (front view).

Simulations were first carried out manually to provide initial estimates of the required somersault angular momentum and timings of the arm and hip movements. Simulated annealing (Goffe et al., 1994) was then used to vary six parameters (comprising the start times and durations of hip movement from side flexion to straight body, and adduction of each arm from $90^{\circ}$ to the side of the body) for the production of tilt and twist (typically using 40,000 simulations). Since there would be some trade-off between maximising tilt and maximising twist depending on the duration used for tilt production, the optimisation criterion was chosen to be that of maximising twist after $1.5 \mathrm{~s}$ without any attempt to remove the tilt. Since the arms were allowed to move through a greater range in the removal of tilt, it was expected that a greater angle of tilt could be coped with for tilt removal. This was verified by running optimisations of reverse simulations that started with the end of flight conditions at time $2.0 \mathrm{~s}$ in which tilt was produced by asymmetrical arm and hip movements (one angle and 8 timing parameters with typically 70,000 simulations) within the permitted ranges, using maximum twist after $0.5 \mathrm{~s}$ as the optimisation criterion. The amount of twist at $1.5 \mathrm{~s}$ in the first optimisation was added to the twist in the reverse simulation at $0.5 \mathrm{~s}$ from the second optimisation to provide an estimate of the maximum twist possible. These timings were used since the body was straight with arms adducted at this time. The maximum twist value was rounded down to the nearest number of half twists: an odd number of half twists for the forward rotating 
double somersault and an even number of half twists for the backward rotating double somersault.

Simulated annealing was then used to find complete performances in which the above twist values were achieved at $2.0 \mathrm{~s}$ along with zero tilt and the required somersault value using a score function that penalised deviations from the final target orientation angles. A total of 16 parameters (using typically 100,000 simulations) were used to vary the asymmetrical arm and hip movements which produced tilt ( 6 parameters) and removed tilt (9 parameters) along with a parameter to adjust the angular momentum value. Additional optimisations were then run to seek solutions with the arms less adducted during the twisting phase, increasing the arm abduction angle by one degree at a time until the target orientation angles failed to be met.

The method of Yeadon (1993d) was used to determine contributions to the production of tilt from arm and hip asymmetries in the two limiting movements. Four cases were considered: asymmetrical arms and asymmetrical hips (the original simulation), arms and hips symmetrical, arms asymmetrical and hips symmetrical, arms symmetrical and hips asymmetrical. The tilt arising from arm asymmetry was taken as the average of the differences in maximum tilt between simulations that differed only in arm symmetry / asymmetry. Since arm symmetry could be achieved in two ways from the original simulation, this involved four comparisons. The tilt arising from hip asymmetry was calculated in a similar way. Contributions to the removal of tilt from arm and hip asymmetries were also calculated using reverse simulations that started with the end of flight conditions at time $2.0 \mathrm{~s}$.

\section{RESULTS}

For the forward rotating double somersault the amounts of twist that could be achieved in the tilt production and tilt removal simulations were 2.70 revolutions and 1.10 revolutions (Table 1 ). As a consequence the forward takeoff limiting movement which used asymmetrical hip movement with wide arms that were adducted sequentially (Figure 1 ) was able to produce $3 \frac{1}{2}$ twists in the second somersault of a piked double forward somersault (Figure 3). A solution was found with the arms abducted $4^{\circ}$ away from rather than adducted $4^{\circ}$ towards the midline of the body in the twisting phase (Figure 4).

Table 1. Maximum twist in tilt production phase and tilt removal phase

\begin{tabular}{|c|c|c|c|c|c|}
\hline & $\begin{array}{c}\text { max } \\
\text { tilt } \\
{[\mathrm{deg}]}\end{array}$ & $\begin{array}{c}\text { twist } \\
1.5 \mathrm{~s} \\
{[\mathrm{rev}]}\end{array}$ & $\begin{array}{c}\text { twist } \\
0.5 \mathrm{~s} \\
{[\mathrm{rev}]}\end{array}$ & $\begin{array}{c}\text { max } \\
\text { twist } \\
{[\mathrm{rev}]}\end{array}$ & $\begin{array}{c}\text { twist } \\
\text { margin } \\
{[\mathrm{rev}]}\end{array}$ \\
\hline forward & $23.9^{\circ}$ & 2.70 & 1.10 & 3.5 & 0.30 \\
\hline backward & $19.0^{\circ}$ & 2.02 & 1.03 & 3.0 & 0.05 \\
\hline
\end{tabular}

Note: twist margin is the difference between the sum of the twists in the two phases and the maximum twist in the limiting movement 


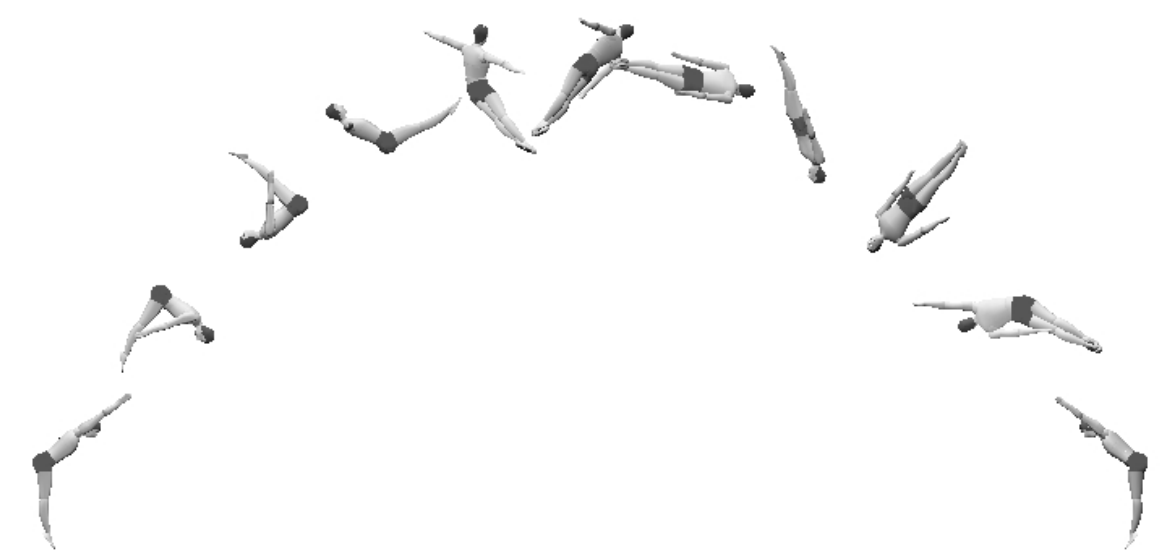

Figure 3. $3 \frac{1}{2}$ twists in the second somersault of a piked double forward somersault (side view).
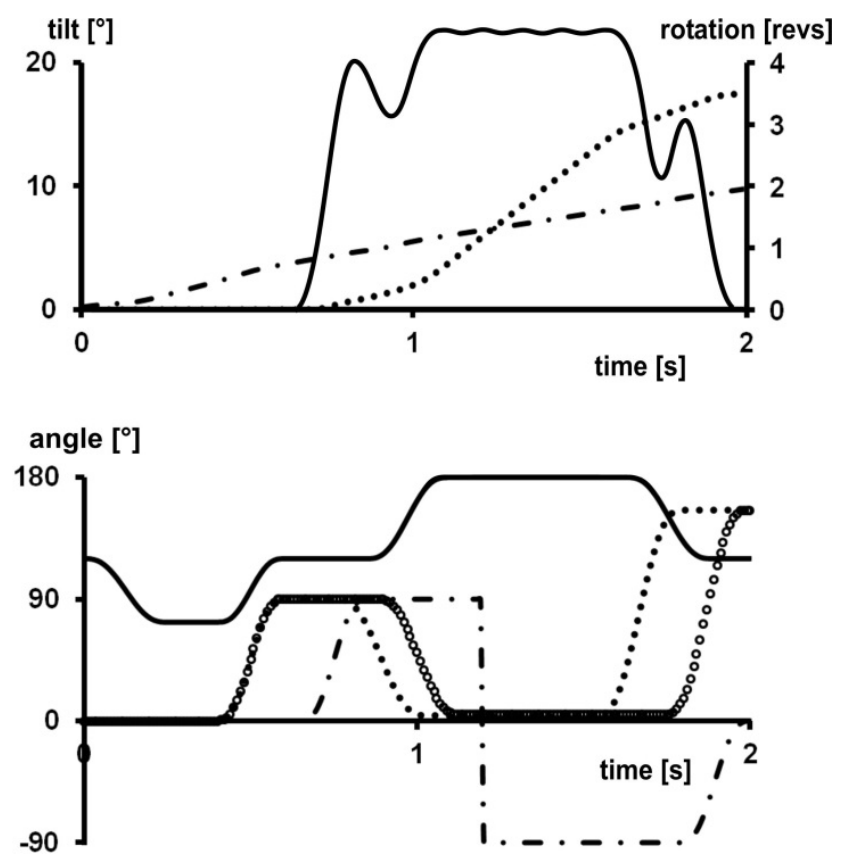

Figure 4. Somersault in revolutions (dashed line), tilt in degrees (solid line) and twist in revolutions (closed circles) during the $3 \frac{1}{2}$ twisting piked double forward somersault (upper graph); hip flexion (solid line), hip hula (dashed line), left arm abduction (open circles), and right arm abduction (closed circles) in degrees (lower graph). Hula angles of $0^{\circ}, 90^{\circ}$ and $-90^{\circ}$ correspond to forward flexion, side flexion over the right hip and side flexion over the left hip.

For the backward rotating double somersault the amounts of twist that could be achieved in the tilt production and tilt removal simulations were 2.02 revolutions and 1.03 revolutions (Table 1 ). As a consequence the backward takeoff limiting movement which also used asymmetrical hip movement with wide arms that were adducted sequentially (Figure 2) was able to produce three twists in the second somersault of a straight double backward somersault (Figure 5). No other solution was found with the arms less adducted than the $4^{\circ}$ that placed them close to the body during the twisting phase (Figure 6). 


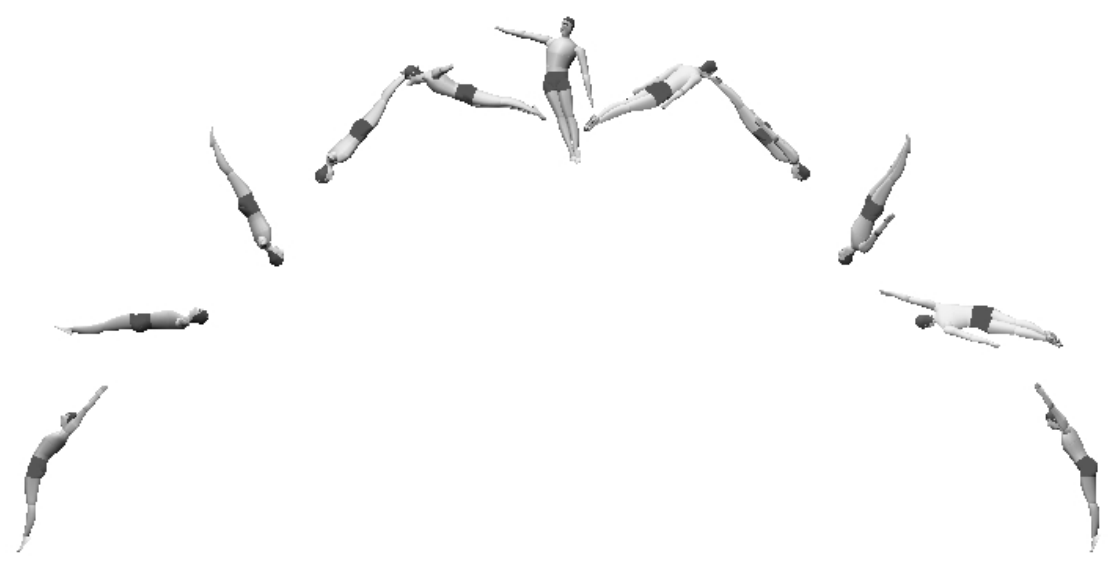

Figure 5. Three twists in the second somersault of a straight double backward somersault (side view).
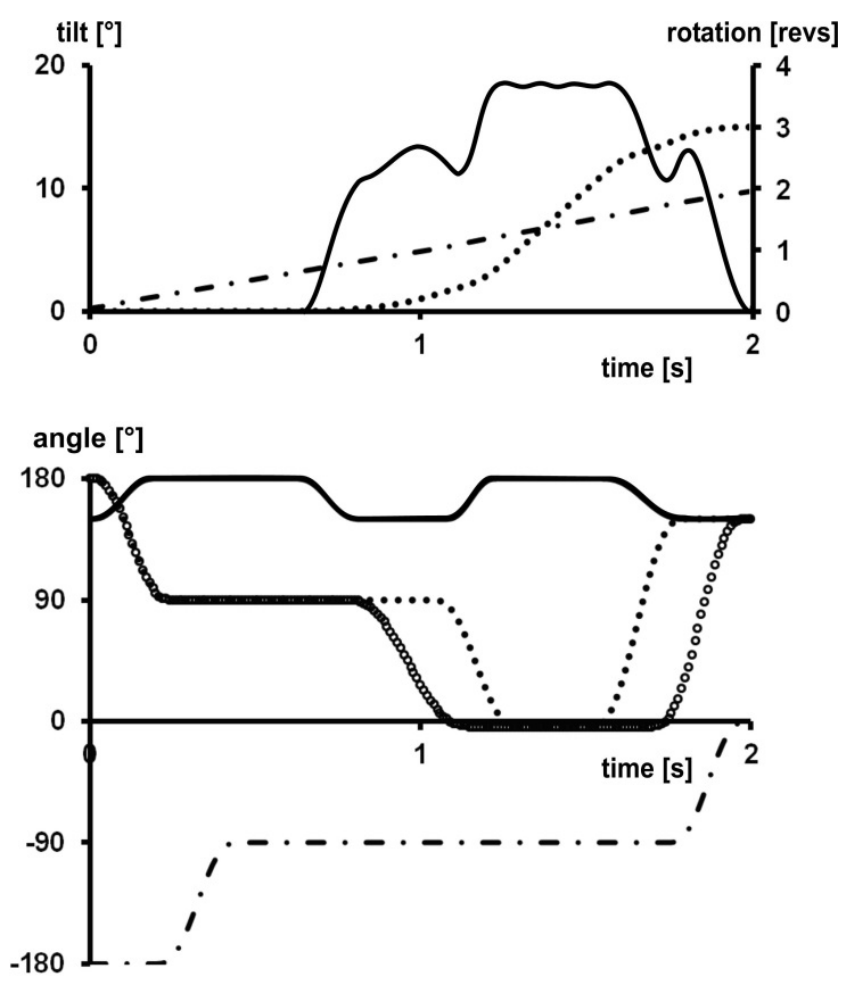

Figure 6. Somersault in revolutions (dashed line), tilt in degrees (solid line) and twist in revolutions (closed circles) during the triple twisting straight double backward somersault (upper graph); hip flexion (solid line), hip hula (dashed line), left arm abduction (open circles), and right arm abduction (closed circles) in degrees (lower graph). Hula angles of $0^{\circ},-90^{\circ}$ and $-180^{\circ}$ correspond to forward flexion, side flexion over the left hip and hyperextension.

Hip asymmetry contributed three times more than arm asymmetry to the production of tilt, on average (Table 2). Hip asymmetry contributed five times more than arm asymmetry to the removal of tilt, on average (Table 3). 
Table 2. Contributions to the production of tilt from arm and hip asymmetries

\begin{tabular}{|l|c|c|c|c|}
\hline & arms & hips & total & actual \\
\hline 3.5 twists & $4.1^{\circ}$ & $18.2^{\circ}$ & $22.3^{\circ}$ & $22.3^{\circ}$ \\
\hline 3.0 twists & $5.9^{\circ}$ & $12.5^{\circ}$ & $18.4^{\circ}$ & $18.4^{\circ}$ \\
\hline
\end{tabular}

Table 3. Contributions to the removal of tilt from arm and hip asymmetries

\begin{tabular}{|l|c|c|c|c|}
\hline & arms & hips & total & actual \\
\hline 3.5 twists & $2.9^{\circ}$ & $19.5^{\circ}$ & $22.4^{\circ}$ & $22.4^{\circ}$ \\
\hline 3.0 twists & $3.8^{\circ}$ & $14.6^{\circ}$ & $18.4^{\circ}$ & $18.4^{\circ}$ \\
\hline
\end{tabular}

\section{DISCUSSION}

The aim of this research study was to determine the twist limits for double somersaults on trampoline with the twist in the second somersault. It was found that $3^{1 / 2}$ twists could be produced in the second somersault of a forward piked double somersault with arms abducted $4^{\circ}$ during the twisting phase and that three twists could be produced in the second somersault of a backward straight double somersault with arms fully adducted.

The forward takeoff limiting movement is known as an Adolf-out fliffus and although it has been performed on trampoline, appreciable twist typically occurs towards the end of the first somersault. In the simulated movement the tilt angle rises from zero in the first somersault to around $22^{\circ}$ early in the second somersault and then falls to zero again at the end of the second somersault (Figure 4). The initial analysis indicated that up to 3.80 twists should be possible and so there should be some margin for variation (Table 1) in the limiting movement with 3.50 twists. This is confirmed by the existence of a solution for the limiting movement with arms abducted by $8^{\circ}$ when twisting compared to the original solution. Since there is some margin for adjustment it is likely that this limiting movement lies just inside the set of achievable movements.

The backward takeoff limiting movement is known as a triple full out and while two twists have been produced in the second somersault of a double backward straight somersault, three twists do not appear to have been achieved. In the simulated movement the tilt angle rises from zero in the first somersault to around $18^{\circ}$ early in the second somersault and then falls to zero again at the end of the second somersault (Figure 6). It is understandable that no solution existed with the arms adducted by less than $4^{\circ}$ since there is little margin for variation (Table 1 ) in the limiting movement. In any performance of a given movement there will be variability 
in execution which may be compensated for later in flight by making adjustments in configuration using feedback control (Yeadon and Hiley, 2014). As there is little margin for making such adjustments it is likely that this limiting movement lies just outside the set of achievable movements.

In the hip asymmetry technique for producing tilt the arms should be held wide for the first quarter twist and then adducted (Yeadon, 1993c) whereas for the arm asymmetry technique sequential arm adduction should be used (Yeadon, 2013). For the production of maximum tilt using a combination of arm and hip asymmetry there will be a trade-off between maintaining wide arms and adducting the arms sequentially before and after the quarter twist position is reached. There will also be a trade-off between maximizing tilt and spending excessive time achieving this. In both limiting movements the sequential arm adduction before and after a quarter twist is apparent (Figures 3,5) leading to increases in tilt (Figures 4, 6).

Although nutation will have a positive effect on increasing the tilt angle during the first quarter twist, it will have a negative effect during the second quarter twist. In order to quantify the net contribution from the nutation effect, a simulation with zero angular momentum in which the twist angle was reset to that of the limiting movement at the end of each time step determined the amount of tilt resulting from the asymmetrical arm and hip movements. The tilt angles obtained in this way were greater than those occurring in the limiting simulations showing that the net effect of nutation was to decrease the tilt angle change both for tilt production and tilt removal.

Since the sum of the tilt angles apportioned to arm and hip asymmetries was equal to the actual tilt in a limiting simulation, these values may be considered as contributions since they are additive. The same is not true if this were to be attempted using twist angles. As might be expected the hip contributions are greater when the hips are more flexed (in the $3^{1 / 2}$ twisting forward double somersault) whereas the arm contributions are unexpectedly smaller (Tables 2,3 ). Presumably this is a consequence of the trade-off in arm movement choice between what is best for asymmetrical hip technique versus asymmetrical arm technique. In each limiting movement both arm and hip asymmetries are evident for the removal of tilt before landing (Figures 3, 5).

The extent to which the various assumptions affect the results of this study will now be considered. In the straight double backward somersault the amount of side flexion during tilt production and removal was restricted to $30^{\circ}$. If more flexion than this were to be used it would detract from the expected extended body configuration and although it would make the twist easier to achieve it would not change the limiting movement. The arm position used in the initiation of twist was restricted to $90^{\circ}$ of arm abduction in order to be more representative of trampolining technique used in a straight double somersault with twist. If more extensive sequential arm movements were permitted, as used in the aerials event of freestyle skiing (Yeadon, 2013), then limiting movements with more twist may be possible. In this case the arm contribution would be greater than in this study. If the requirement of a symmetrical arm configuration at landing were to be relaxed, this would make the removal of tilt easier but would not change the limiting movements.

The lower bounds placed on the durations of arm and hip angle changes were based on actual performances and may possibly overestimate the minimum time needed for a joint movement. The effect of this was investigated by reducing all lower bounds on durations by $20 \%$ and the effects on optimisations determined. Tilt production increased by only $0.1^{\circ}$. The twist margin for the forward twisting 
movement increased from 0.30 to 0.48 twists. The twist margin for the backward twisting movement increased from 0.05 to 0.23 twists. The limiting movements did not therefore change in either case. Although such timing changes may make the limiting movements easier, the effects are limited due to the relationship between somersault rate, tilt angle and twist rate (Yeadon, 1993a; Mikl and Rye, 2016). The above considerations also imply that increasing the time of flight from $2.0 \mathrm{~s}$ to $2.5 \mathrm{~s}$ would not change the limiting movements. Thus the value of $2.0 \mathrm{~s}$ for the flight time is not a limitation of the study.

The segmental inertia parameters of one male individual were used in the determination of the limiting movements. For comparison the inertia parameters of a female trampolinist and a male springboard diver were used to determine the two limiting movements. It was found that the same limiting movements were obtained in both cases with arm adduction angles in the twisting phase less than $2^{\circ}$ different from those reported in this study. A particular individual athlete's segmental inertias may result in the limiting movements having less twist (Mikl, 2016). The same may also be true of a particular individual's strength and precision limits. The aim of this study, however, was to determine the limits for elite trampolinists.

In order that a given movement can be performed consistently there has to be scope for adjusting each performance in order to control the twist as in Yeadon and Hiley (2014). For the forward takeoff limiting movement there is scope for adjustment since there is a range of $8^{\circ}$ of arm abduction available. For the backward takeoff limiting movement there is little room for adjustment since the arms are fully adducted during the twisting phase with negligible range of arm abduction available. In this study the criteria for attaining the final target angles of somersault, tilt and twist were that they should be met exactly. If some tolerance were to be introduced for meeting the target angles, this would not change the limiting movements but would allow a greater range of arm abduction angle and hence more scope for adjusting an individual performance.

It can be concluded that on trampoline the limiting movements for late twisting double somersaults are $3 \frac{1}{2} 2$ twists in a forward piked double somersault and three twists in a backward straight double somersault with the twist occurring in the second somersault in each case.

\section{CONFLICT OF INTERSEST STATEMENT}

There are no issues of conflict of interest arising from the personal or professional associations of the authors.

\section{REFERNCES}

Brüggemann, G-P., Arampatzis, D., 1993. Simulation of a quadruple backward somersault dismount from the high bar. In: Bouisset S, Métral S, Monod S (eds) Abstracts, International Society of Biomechanics XIV ${ }^{\text {th }}$ Congress, pp. 222-223, Paris, 4-8 July 1993.

Goffe, W.L., Ferrier, G.D., Rogers, J., 1994. Global optimization of statistical functions with simulated annealing. Journal of Econometrics 60, 65-99.

Hiley, M.J., Yeadon, M.R., 2003. Optimum technique for generating angular momentum in accelerated backward giant circles prior to a dismount. Journal of Applied Biomechanics 19, 119-130. 
Hiley, M.J., Yeadon, M.R., 2005. Maximal dismounts from high bar. Journal of Biomechanics 38, 2221-2227.

Hiley, M.J., Jackson, M.I., Yeadon, M.R., 2015. Optimal technique for maximal forward rotating vaults in men's gymnastics. Human Movement Science 41, 1730-1735.

King, M.A., Yeadon, M.R., 2004. Maximising somersault rotation in tumbling. Journal of Biomechanics 37, 471-477.

Mikl, J., 2016. All spun out: Limits of aerial techniques when performing somersaults. PhD thesis, University of Sydney. http://hdl.handle.net/2123/15375.

Mikl, J., Rye, D.C., 2016. Twist within a somersault. Human Movement Science 45, 23-39.

Yeadon, M.R., 1990a. The simulation of aerial movement - I: The determination of orientation angles from film data. Journal of Biomechanics 23, 59-66.

Yeadon, M.R., 1990b. The simulation of aerial movement - II: A mathematical inertia model of the human body. Journal of Biomechanics 23, 67-74.

Yeadon, M.R., 1990c. The simulation of aerial movement - III: The determination of the angular momentum of the human body. Journal of Biomechanics 23, 7583.

Yeadon, M.R., 1993a. The biomechanics of twisting somersaults. Part I: Rigid body motions. Journal of Sports Sciences 11, 187-198.

Yeadon, M.R., 1993b. The biomechanics of twisting somersaults. Part II: Contact twist. Journal of Sports Sciences 11, 199-208.

Yeadon, M.R., 1993c. The biomechanics of twisting somersaults. Part III: Aerial twist. Journal of Sports Sciences 11, 209-218.

Yeadon, M.R., 1993d. The biomechanics of twisting somersaults. Part IV: Partitioning performance using the tilt angle. Journal of Sports Sciences 11, 219-225.

Yeadon M.R., 2013. The limits of aerial twisting techniques in the aerials event of freestyle skiing. Journal of Biomechanics 46, 1008-1013.

Yeadon, M.R., Atha, J., Hales, F.D., 1990. The simulation of aerial movement - IV: A computer simulation model. Journal of Biomechanics 23, 85-89.

Yeadon, M.R., Hiley, M.J., 2014. The control of twisting somersaults. Journal of Biomechanics 47, 1340-1347. 